\title{
Motivación y valores; herramientas que posibilitan la trayectoria académica de los preparatorianos
}

\section{Motivation and values; tools that enable the academic trajectory of preparatorians}

PÉREZ-CASTRO, Francisco Isaí $\dagger *$ ， TERRAZAS-MEDINA， Tamara Isabel, MARTINEZCÁRDENAS, Juana María y LOPEZ-NIEBLA, Rosa María

Instituto de Enseñanza Abierta, Universidad Autónoma de Coahuila

ID 1 ${ }^{\text {er }}$ Autor: Francisco Isaí, Pérez-Castro / ORC ID: 0000 - 0002 - 6779 - 7881, CVU CONACYT ID: 929950

ID 1 ${ }^{\mathrm{er}}$ Coautor: Tamara Isabel, Terrazas-Medina / ORC ID: 0000 -0002-6581 -190X, CVU CONACYT ID: 929839

ID $2^{\text {do }}$ Coautor: Juana María, Martínez-Cárdenas / ORC ID: 0000-0003-1004 9652, Research ID Thomson: X-2370-2018, CVU CONACYT ID: 949979

ID $3^{\text {er }}$ Coautor: Rosa María, López-Niebla / CVU CONACYT ID: 949982

\section{Resumen}

La presente investigación se trabajó bajo la norma cualitativa de diseño flexible, siendo interpretativa y diacrónica, donde se ejecutó bajo una entrevista semi estructurada donde participaron jóvenes de 15 y 17 años de edad. Los procesamientos de información se trabajaron en el programa Atlas Ti para obtener evidencias sobre la interrogante de investigación. Los resultados de la investigación más relevantes se enfocan hacia la motivación docente como parte fundamental en la vida de los estudiantes logrando sacar de ellos su esencia a través de herramientas que posibilitan el proceso de enseñanza. El objetivo primordial radica en el análisis y reflexión sobre el Proyecto a Favor de la Convivencia Escolar de la SEP que busca favorecer la convivencia inclusiva, democrática y pacífica en las escuelas, a partir de acciones que contribuyan al trabajo académico de docentes y estudiantes en el aula, a partir de la perspectiva "aprender a aprender y aprender a convivir".

Motivación, Valores, Estudiantes

\begin{abstract}
This research was carried out under the qualitative standard of flexible design, being interpretive and diachronic, where it was executed under a semistructured interview where young people aged 15 and 17 participated. The information processing was worked on the Atlas Ti program to obtain evidence on the research question. The most relevant research results focus on teacher motivation as a fundamental part of the students' lives, achieving their essence from them through tools that enable the teaching process. The primary objective lies in the analysis and reflection on the Project for the School Coexistence of the SEP that seeks to promote inclusive, democratic and peaceful coexistence in schools, based on actions that contribute to the academic work of teachers and students in the classroom, from the perspective "learn to learn and learn to live together".
\end{abstract}

Motivation, Values, Students

Citación: PÉREZ-CASTRO, Francisco Isaí, TERRAZAS-MEDINA, Tamara Isabel, MARTINEZ-CÁRDENAS, Juana María y LOPEZ-NIEBLA, Rosa María. Motivación y valores; herramientas que posibilitan la trayectoria académica de los preparatorianos. Revista de Filosofía y Cotidianidad. 2019, 5-17: 12-16

$\dagger$ Investigador contribuyendo como primer Autor. 


\section{Introducción}

El tema de la motivación ha sido interés de muchos investigadores desde tiempo atrás enfocándose al conocer del hombre, el saber cómo piensa, cuáles son sus necesidades, por qué actúa de determinada manera, por qué prefiere o no ciertos objetos, cómo y hacia dónde se proyecta. Lo que ha conllevado al ámbito educativo, ser el centro de observación y aplicación de esta temática.

En el proceso de enseñanza aprendizaje la motivación juega un papel importante en la actuación del individuo, al igual que en todas las esferas de actuación del hombre. La motivación ayuda al logro de los objetivos de dicho proceso. Los estudiantes realizan una u otra actividad satisfactoriamente si el nivel de motivación hacia la misma es adecuado.

Por otro lado, la familia y la escuela son un marco referencial para la incorporación de un ser humano a la sociedad; pero, este marco se encuentra a los pies de los cambios impuestos por transformaciones diversas, como el gobierno, que han de asumir ambas instituciones, familia y escuela, si quieren responder a su tarea educativa y socializadora.

El objetivo principal de este trabajo es explorar, pero a la vez reflexionar sobre la motivación y el uso de valores en el salón de clase enfocado a jóvenes de bachillerato. Esta propuesta que se presenta nace de la necesidad de dar solución a las siguientes aseveraciones que el Sistema Educativo Mexicano colocó para ir en pro de las exigencias sociales y psico educativas que nuestro país requiere.

Dichas aseveraciones se engloban como expectativas de la educación en México, ejemplo de ellas se ubica en la educación media superior, donde los jóvenes aprendan a regular sus emociones, a trabajar en colaboración, resolver conflictos mediante el diálogo y a respetar las reglas de convivencia en el aula, en la escuela y fuera de ella.

Es lo anterior lo que se toma como fundamento, además de la praxis de la teoría que conforma este trabajo, para proponer alternativas de acción dirigidas a los personajes que se encuentran dentro del círculo de desarrollo de un joven como lo son, los docentes, los padres de familia y otros individuos cercanos a éstos.

\section{Metodología a desarrollar}

En primer lugar, se elaboró una entrevista de 12 preguntas para conocer la percepción que tenían los estudiantes acerca de la motivación y los valores en el salón de clase. Dos de ellas se refería a preguntas generales, 3 abiertas y 7 cerradas.

Esta entrevista fue realizada en un primer momento con un compañero de clase y con base a un juicio de valor, donde el docente estuvo presente, se decidió pasar a la segunda fase.

El día 11 de abril de 2019 se procedió a entrevistar a 10 jóvenes de los cuales se pudo extraer información relevante mas no suficiente por lo que se decidió hacer una observación, la cual consistía en analizar cada uno de los detalles de una clase, la cual correspondía al grupo donde pertenecían los jóvenes a quienes entrevistamos. Misma tuvo efecto el 18 de abril del mismo año.

La información que se recabó fue llenada con base a una ficha de observación. Las entrevistas se vaciaron en Word y se trabajó bajo el software Atlas Ti para poder trabajar la información y presentarla bajo un análisis subjetivo sustentado en las respuestas de los participantes y representada en redes axiales.

\section{Marco Teórico}

Los valores comienzan desde una edad muy temprana en el seno familiar en un primer momento; la sociedad es quien catapulta y los fortalece, pero la escuela es quien tiene la responsabilidad de transformar y formar los valores esenciales para el bien común.

El proceso de enseñanza-aprendizaje, donde participan tanto los docentes como los estudiantes, siempre se forma y desarrollan valores considerados como el marco conceptual de lo que el individuo desea o relaciona lo deseable con lo indeseable. Desde tiempos remotos se han visualizado definiciones acerca de los valores, cada vez más complejas y difíciles de aterrizar a la práctica, una definición operativa y que conceptualiza la esencia de este conjunto de características que nos definen como individuos es (Herrera, M, 2009) quien nos dice que los valores son la relación entre la realidad objetiva y los componentes de la personalidad, lo que se expresa a través de conductas y comportamientos. 
Por lo que se puede decir que en el actuar pedagógico, se trata de alcanzar comportamientos como resultado de aprendizajes significativos, colaborativos y constructivistas en el ámbito racional y emocional. Actualmente, estamos viviendo una crisis de valores, expresada en una gran dificultad para tolerar la diferencia, incapacidad para ser solidario, ausencia del respeto ante las necesidades de los demás la falta de responsabilidad y de compromiso, la indiferencia ante el dolor de los otros, es por esto que los (López, M. 2003) valores y los principios son parte fundamental del desarrollo del ser humano porque constituyen guías de interacción y de convivencia con los demás.

El maestro es una pieza fundamental en el entorno de la persona que se encuentra en el proceso de formación, es la escuela donde surgen los primeros contrastes y muchas veces los primeros conflictos entre lo que el niño vive en su casa y obviamente en la institución. Marti, J. (1975) nos dice que educar es depositar en cada hombre toda la obra humana que le ha antecedido: es hacer de cada hombre resumen del mundo viviente hasta el día en que vive. Es ponerlo a nivel de su tiempo, para que flote sobre él y no dejarlo debajo de su tiempo, con lo que no podrá salir a flote; es preparar al hombre para la vida.

Los docentes apoyan la idea de trabajar en un salón de clases bajo un ambiente donde la interacción entre el maestro y los alumnos sea favorable y deseable dentro del aula y la comunidad estudiantil en general; Kepowicz (2007) apunta según los resultados de su estudio que estudio que de esta manera se desarrollará la educación constructivista y dejar en menor medida enfocarse a los aspectos de conducta del estudiante.

Proveer a los jóvenes de un ambiente escolar donde no solamente recibe órdenes por parte de un maestro para que realice tareas sobre los contenidos expuestos, sino brindarles la oportunidad de experimentar situaciones en las que tengan un crecimiento personal y cultural a través de la interacción y relaciones de sentido entre ellos y sus compañeros e incluso familiares; de esta manera refiere Kepowicz (2007) los estudiantes tendrá la posibilidad de cambiar junto con su contexto las perspectivas individuales o grupales de una realidad especifica.
No se trata solamente de adaptar al individuo en el ambiente, sino que son el individuo y el ambiente los que se modifican mutuamente, en una interacción dinámica en la cual las personas construyen sus ideas sobre su medio físico, social y cultural.

Por otro lado, existen diversos conceptos para la motivación, en este caso presentaremos la definición proporcionada por Bacigalupe \& Mancini (2012) que define a la motivación como a una variedad de factores fisiológicos y neurales que inician, sostienen y dirigen el comportamiento en relación con el ambiente del sujeto. (...), los estados motivacionales se asocian a cambios cerebrales e incluyen el medio del sujeto: el ser humano es un organismo abierto, en permanente interacción con su ambiente.

Es por esto que la motivación se ha subdividido en dos aspectos, esto es como la motivación interna y externa, la motivación interna es la que tiene como objetivo la neurociencia refiriendo que no solo los factores tales como las metas u objetivos que un individuo necesita alcanzar repercuten en su motivación; sino que los conocimientos que éste tenga para alcanzarlos influirán notoriamente en la estimulación interna que tenga, es decir, la motivación que lo impulse a llevarlos a cabo.

Una persona que tiene como fin un logro en su vida y que para lograrlo esta consiente que eso significa un esfuerzo y una disciplina consigo mismo para lograrlo, le será más fácil experimentar la motivación en su proceso de aprendizaje. Ospina (2006) nos dice que los alumnos intrínsecamente motivados toman el aprendizaje en sí mismo como una finalidad y los incentivos para aprender se encuentran en la propia tarea, por lo cual persiguen la resolución de ella y "tienden a atribuir los éxitos a causas internas como la competencia y el esfuerzo".

Ospina (2016) apunta que, de manera opuesta, la motivación extrínseca "es el efecto de acción o impulso que producen en las personas determinados hechos, objetos o eventos que las llevan a la realización de actividades", pero que proceden de fuera. De esta manera, el alumno extrínsecamente motivado asume el aprendizaje como un medio para lograr beneficios o evitar incomodidades. Por ello, centra la importancia del aprendizaje en los resultados y sus consecuencias. 
Lo anterior mencionado, hace alusión a lo que según Barrios Gómez (2013) señala como característica del Plan de estudios; su orientación hacia el desarrollo de actitudes, prácticas y valores sustentados en los principios de la democracia: el respeto a la legalidad, la igualdad, la libertad con responsabilidad, la participación, el diálogo y la búsqueda de acuerdos; la tolerancia, la inclusión y la pluralidad, así como una ética basada en los principios del Estado laico, que son el marco de la educación humanista y científica que establece el Artículo Tercero Constitucional. SEP Plan de Estudios (2011, p.30).

Buscar una educación en valores con el objetivo de potencializar el uso de ellos para reducir la gran crisis valoral presentes en las generaciones del hoy, sienta las bases para que los pensadores contemporáneos sugieran criterios para la elaboración de modelos educativos con fundamentos que van desde las normas constitucionales y las nuevas perspectivas científicas que sugieren un actuar humanista.

Es labor de cada ciudadano llámese docente, estudiante, carpintero, político, etcétera hacer que le cambio de una sociedad carente de valores a una sociedad consiente de ellos en las generaciones presentes y futuras sea un sueño inalcanzable para convertirse en un hecho tangible a través de una sociedad feliz con una calidad de vida ejemplar.

\section{Resultados}

En la figura 1 se describe la relación que muestra la motivación y los valores en el aula escolar de los alumnos de bachilleres Dr. Mariano Narváez; refiriendo como los principales detonadores que motivan a estos estudiantes a la obtención de buenas calificaciones o puntos extras por parte de los docentes para de este modo recibir el alago de sus padres, quienes les otorgan premios y regalos por haber destacado en el semestre escolar.

Los jóvenes al experimentar un ambiente donde se les reconoce se sienten animados, con un grado de confianza que les permite desarrollar con libertad y compañerismo la ejecución de las actividades escolares y con deseos de lograr las expectativas de vida que poseen; destacando como fuente principal de la motivación a los padres de éstos.

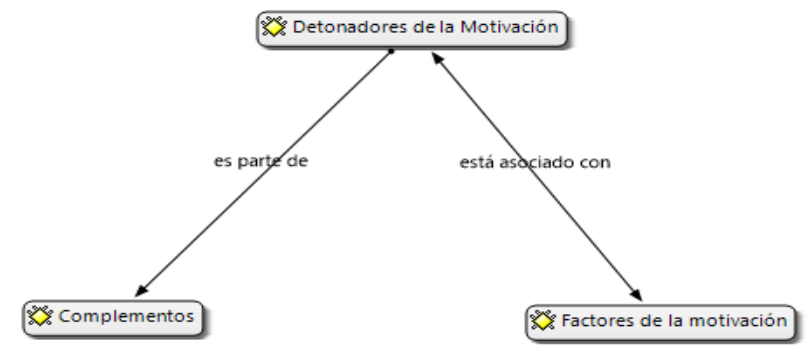

Figura 1 Red Axial de los detonadores de la motivación en el salón de clase

En la figura 2 se evidencia la relación que muestra la motivación y los valores en el aula escolar de los alumnos dl bachillerato Dr. Mariano Narváez T.M se puede observar como eje central el actuar pedagógico demostrado así con el cuestionamiento de temáticas, el aconsejar y la retroalimentación. A causa de esto se abre paso a la utilización de valores, que a través de la motivación docente como lo es el incentivar, integrar e incitar a sus alumnos al estudio se logre enriquecer esta red donde el docente es quien dirige y es responsable de su sentir hacia los demás.

Con lo anterior, se enmarca la motivación docente dentro del actuar pedagógico de manera que las técnicas y procedimientos llevan consigo la intención valores que repercuten y transforman la conducta del estudiante de manera que juntos logran crear un espacio de dialogo a través de vivencias personales.

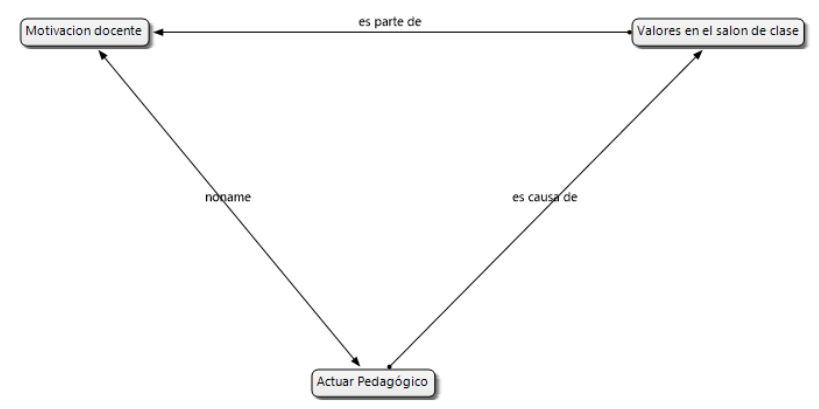

Figura 2 Red Axial del actuar pedagógico del docente en el salón de clase

La tabla 1 muestra las categorías que se vinculan con la motivación del docente, donde las principales herramientas que utilizan son, el incentivar, la confianza y la integración. 
El incentivar se refleja con frases apremiantes como ¡Muy Bien! o $¡$ Gracias por tu aportación!, la confianza se da cuando el docente conoce y se acerca a lo que sus estudiantes piensan a través de sus historias de vida y la integración donde su misión sea lograr que los estudiantes trabajen colaborativa y cooperativamente estando en un espacio propiciado por el docente donde se respire tranquilidad y se sientas cómodos.

Por lo anterior se puede con concluir que la motivación docente es parte fundamental en la vida de los estudiantes logrando sacar en ellos su esencia a través de herramientas que posibilitan el proceso de enseñanza y de organización.

\begin{tabular}{ccc}
\hline Unidad de Análisis & \multicolumn{1}{c}{ Categoria } & \multicolumn{1}{c}{ Subcategoria } \\
\hline & Incentivar & $\begin{array}{l}\text { Con la frase jMuy Bien! } \\
\text { ¡Gracias por tu aportación! }\end{array}$ \\
\cline { 2 - 3 } Motivación docente & Confianza & $\begin{array}{l}\text { Conoce lo que piensan los } \\
\text { estudiantes a través de } \\
\text { sus vivencias. }\end{array}$ \\
\cline { 2 - 3 } & Integración & $\begin{array}{l}\text { Logra que los estudiantes } \\
\text { trabajen en equipos y que } \\
\text { todos se sientan cómodos }\end{array}$ \\
& & \\
\hline
\end{tabular}

Tabla 1 Análisis del concepto motivación del docente

\section{Conclusiones}

Con lo que respecta se puede decir que la motivación aportará resultados en el aprendizaje de los estudiantes, debido a que dentro de ella se tiene la necesidad de conocer y aprender cosas nuevas y atractivas que enriquezcan sus nociones y se tengan formas de aprender y estrategias para una mejor educación.

Cada una de las estrategias que se aplican dentro del aula será de valor y apoyo para una mejor comprensión del tema que se esté impartiendo en un periodo de clase, por tanto, es necesario que el docente implemente técnicas que colaboren en el desarrollo de la clase y por ende en el aprendizaje de los estudiantes de preparatoria.

Es importante saber que las estrategias siempre se tienen que realizar de acuerdo a las habilidades y debilidades de los alumnos, ya que de ellas depende captar la atención del estudiante de tal modo que muestre interés por lo que el docente le está impartiendo en ese momento.
Si bien esta labor se complementa con los valores donde muchas de las personas tienen la idea equivocada al pensar que los encargados de inculcar los valores en los estudiantes son los docentes. Juzgan y hablan más de los docentes sin darse cuenta que en realidad los padres de familia son los verdaderos responsables, pues son los encargados de inculcar los valores en los estudiantes y por parte de los docentes está el reforzarlos día a día, sin embargo algunas ocasiones estos dos se deslindan de esta obligación con la ideología de "en la escuela se los enseñan" o "sus padres deben hacerlo" lo cual es completamente erróneo pues se necesita apoyo y responsabilidad por parte de los dos para obtener mejores resultados.

\section{Referencias}

Bacigalupe, M. \& Mancini, V. (2012) Motivación y Aprendizaje: contribuciones de las neurociencias a la fundamentación de las intervenciones educacionales. Universidad Iberoamericana. DIDAC p. 44, 46.

Barrios, E. (2013) La educación en valores factor fundamental para la mejora de la calidad educativa y de la calidad de vida de los mexicanos. Pálido de Luz. Consultado el día 30 de Marzo de 2019 en: http://palido.deluz.mx/articulos/1095

Díaz Barriga, A. (2006). La educación en valores: Avatares del currículum formal, oculto y los temas transversales. Revista Electrónica de Investigación Educativa, 8 (1). Consultado el día 01 de mayo de 2019 en: http://redie.uabc.mx/vol8no1/contenidodiazbarriga $2 . h t m l$

Gavieria, E y Fernandez, I (2006) La motivación social. En A. Gómez, E. Gaviria y I. Fernández (Coords): Psicología social, pp.33-75. Madrid: Editorial Sanz y Torres.

Gómez, C y Gargallo, B. (2008) Construcción humana y procesos de estructuración. Pr. Juan Escames Sánchez. Universidad de Valencia.

Ospina, J. (2006) La motivación, motor del aprendizaje. Revista Ciencias de la Salud. Consultado el 21 de febrero de 2016 en: http://redalyc.org/articulo.oa?id=56209917 